\title{
Um modelo de Ambiente Virtual de Aprendizagem de Conceitos Geométricos utilizando Suportes Pedagógicos e o GeoGebra
}

\author{
Fábio Paraguaçu ${ }^{1}$ \\ Instituto de Computação/Universidade Federal de Alagoas IC/UFAL \\ Fabioparagua200@gmail.com \\ Terence Coelho Lopes de Magalhães ${ }^{2}$ \\ Instituto de Computação/Universidade Federal de Alagoas IC/UFAL \\ Terelan5@ hotmail.com
}

Resumo: A ciência da aprendizagem tem apontado para a necessidade da implantação de pedagogias ativas para a aprendizagem dos conceitos geométricos. Nesta perspectiva, esse trabalho descreve a modelagem da aprendizagem de conceitos geométricos através da resolução de problemas em um ambiente virtual de aprendizagem utilizando suporte pedagógico e um micromundo em geometria, o GeoGebra, favorecendo a compreensão e a construção do raciocínio geométrico. A metodologia de pesquisa utilizada foi o "Estudo de Caso", onde foi observado o comportamento dos estudantes nos experimentos propostos. A pesquisa mostra avanços na aprendizagem dos estudantes, e mostra a evolução dos estudantes na resolução de problemas e compreensão dos conceitos geométricos.

\section{Palavras-chave: Suporte pedagógico, Ambiente Virtual de Aprendizagem, GeoGebra.}

\section{A Virtual Environment Model for Learning Geometric Concepts Using Pedagogical Supports and the GeoGebra}

\begin{abstract}
The science of learning has pointed to the need of the implantation of active pedagogies for the learning of the geometric concepts. In this perspective, this work describes the modeling of learning of geometric concepts through problem solving in a virtual learning environment using pedagogical support and a microworld in geometry, GeoGebra, favoring the understanding and construction of geometric reasoning. The research methodology used was the "Case Study", where the behavior of the students in the proposed experiments was observed. The research shows advances in student learning, and shows students' evolution in problem solving and understanding of geometric concepts.
\end{abstract}

\section{Keywords: Pedagogical support, Virtual Learning Environment, Geogebra.}

\section{INTRODUÇÃO}

A proposta deste artigo é apresentar um modelo computacional para auxiliar os estudantes na aprendizagem de conceitos geométricos por meio da resolução de problemas e da construção de figuras geométricas.

Os estudantes apresentam dificuldades na aprendizagem de conceitos geométricos, fato abordado por muitos autores, Gravina (1996/1999), Valente (2004) e nesse sentido atribuem as dificuldades na aprendizagem a alguns fatores como ensino não individualizado, a heterogeneidade de raciocínios e a variabilidade no ritmo de aprendizagem (Valente,2004).

\footnotetext{
${ }^{1}$ Professor Doutor do Programa de Pós- Graduação em Modelagem Computacional do Conhecimento em EducaçãoIC/UFAL ${ }^{2}$ Mestranda do Programa de Pós- Graduação em Modelagem Computacional do Conhecimento em Educação do IC/UFAL. Bolsista FAPEAL/CAPES.
} 
Segundo Bayer (2004), existe uma grande preocupação entre professores e matemáticos em relação ao ensino deste conteúdo. A busca de novas formas e práticas pedagógicas para se resgatar o ensino de Geometria com qualidade tem sido destaque em trabalhos de pesquisadores em todo o mundo.

Neste artigo serão usadas, como suporte pedagógico, as fases de resolução de problemas de Polya (1975), compreender o problema, tem que se perceber claramente o que é necessário, observar a relação entre a incógnita, os dados para ter a ideia de resolução, estabelecimento de um plano, execução do plano, e fazer um retrospecto da resolução, revendo-a e discutindo-a.

A aprendizagem de conceitos geométricos será baseada na teoria da Assimilação Significativa de Ausubel (2000) e na Zona de Desenvolvimento Proximal de Vygotsky Sloczinski(2010).

Segundo Barrows (1980), a aprendizagem baseada em problemas tem dois postulados fundamentais. O primeiro é que a aprendizagem através da resolução de problemas é muito mais eficaz para criar na mente do estudante o conhecimento utilizável no futuro em detrimento do que é tradicional a aprendizagem baseada em memorização. $O$ segundo é que as habilidades mais importantes são obtidas através da resolução de problemas, não em habilidades de memória.

E nesse sentido, Perrenoud (2000) reforça que faltam aos estudantes alguns conhecimentos básicos em campos específicos da Matemática, por exemplo, que foram estudados de forma descontextualizada e que quando precisam ser resgatados por ele para sua utilização na vida prática, acabam por não fazer correlação consciente entre a matéria dada e a competência exigida.

Este artigo propõe um ambiente virtual de aprendizagem (AVA) com foco na resolução de problemas utilizando suportes pedagógicos. Para compreensão e interpretação das figuras geométricas, é utilizado o GeoGebra, incorporado como aplicação no Ambiente Virtual de Aprendizagem.

\section{Os Suportes Pedagógicos e a Teoria da assimilação significativa}

Segundo Illeris (2003) a aprendizagem assimilativa ou por adição ocorre quando o novo elemento é incorporado como uma adição constante a um esquema ou padrão que já estava estabelecido, ao que já foi aprendido. Ocorre em todos os contextos onde o aprendiz desenvolve suas capacidades gradualmente.

De acordo com o autor, os resultados da aprendizagem se caracterizam por estarem ligados ao esquema ou padrão em questão, de modo que seja relativamente fácil de recordálos e aplicá-los quando se está mentalmente orientado para o campo em foco, por exemplo, uma disciplina escolar, enquanto pode ser difícil acessá-los em outros contextos.

Uma das metas do processo de aprendizagem e ensino é estimular o aprendiz a ser autônomo, isto é, sujeito do seu próprio aprender (PORTILHO, 2011).

De acordo com Ausubel (2000) a Teoria da Assimilação explica a forma como se relacionam de modo seletivo, na fase de aprendizagem, novas ideias potencialmente significativas do material de instrução com ideias relevantes, e, também, mais gerais e inclusivas, bem como mais estáveis, existentes e ancoradas na estrutura cognitiva.

De acordo com o The Glossary of Education ${ }^{1}$ na educação, scaffolding refere-se a uma variedade de técnicas de instrução usados para mover os alunos progressivamente em direção à compreensão mais forte e, em última instância uma maior independência no processo de aprendizagem. Os professores fornecem níveis sucessivos de apoio temporário que ajudam os alunos a alcançarem níveis mais elevados de compreensão e habilidade de aquisição que eles não seriam capazes de alcançar sem assistência.

${ }^{1}$ http://edglossary.org 


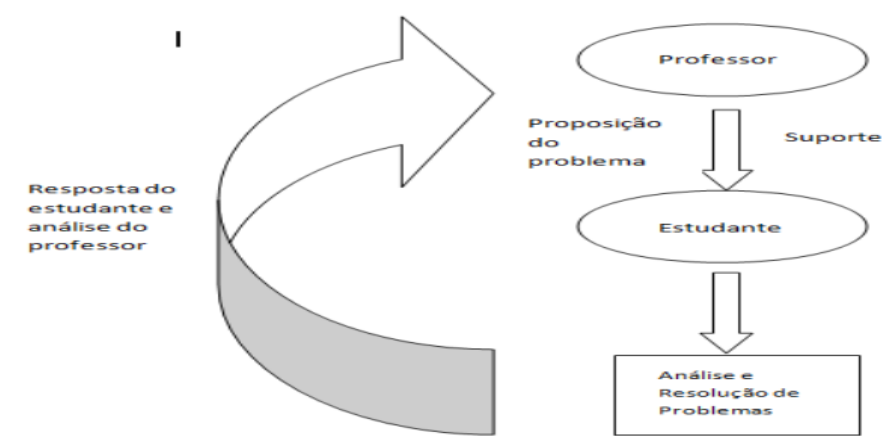

Figura 1 - Estrutura de suporte pedagógico

Fonte: Arquivo pessoal

Segundo Reiser (2014), as ferramentas podem moldar o desempenho dos alunos e a compreensão da tarefa em relação ao conteúdo disciplinar chave e às estratégias e, assim, problematiza o conteúdo, forçando os alunos a se envolverem com a complexidade, tais ferramentas de suporte tornam este trabalho mais produtivo e promove oportunidades de aprendizagem.

De acordo com Reiser (2014), scaffolding é uma estratégia-chave no aprendizado cognitivo, no qual os alunos podem aprender assumindo cada vez mais responsabilidade na resolução de problemas complexos com a estrutura e a orientação de professores.

A progressividade em que o suporte é retirado demonstra a aprendizagem do estudante e a autonomia na resolução de problema.

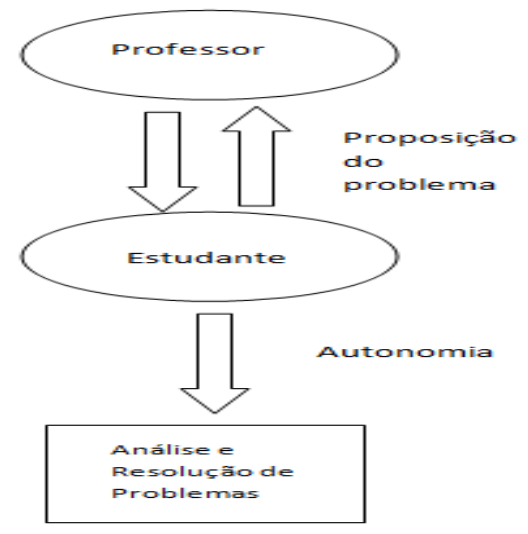

Figura 2 - Estrutura de progressividade na resolução dos problemas.

Fonte: Arquivo pessoal

Segundo Polya (1975), as fases de resolução de problemas são:

- Compreensão do problema - É preciso compreender o problema, identificar a incógnita, os dados e a condicionante.

O autor utiliza questionamentos que auxiliam a compreensão: É possível satisfazer a condicionante?A condicionante é suficiente para determinar a incógnita?Ou é insuficiente?Ou redundante?Ou contraditória?

É indicado pelo autor que a figura seja traçada e que se adote uma notação adequada.

- Estabelecimento de um plano - Já viu o problema ou um problema correlato? Conhece um problema que pode ser útil?

Considerando a incógnita, ele auxilia o estudante a procurar um problema que tenha a mesma incógnita ou outra semelhante. 
Considerando um problema correlato e já antes resolvido, é possível utilizá-lo? Utilizar o seu resultado?O seu método?Deve-se introduzir algum elemento auxiliar para tornar possível a sua utilização? É possível reformular o problema?

- Execução do plano - É possível verificar se ele está correto?É possível demonstrar que ele está correto?

- Retrospecto - É possível verificar o resultado?É possível verificar o argumento?É possível chegar ao resultado por um caminho diferente?É possível perceber isso num relance?É possível utilizar o resultado ou o método em algum outro problema?

De acordo com Polya (1975), o problema deve ser proposto ao estudante e o professor deve ajudá-lo a resolver. O estudante deve ser levado ao questionamento, através das indagações, estimulando a elaboração de estratégias para a resolução do problema.

\section{GeoGebra e a Aprendizagem de Conceitos Geométricos}

Os conceitos geométricos constituem parte importante do currículo de Matemática no ensino fundamental, porque, por meio deles, o estudante desenvolve um tipo especial de pensamento que lhe permite compreender, descrever e representar, de forma organizada, o mundo em que vive (PCN, 1998).

Os conceitos de geometria possuem regras, o desenho não é meramente um esboço, são as características das figuras que ajudam na resolução do problema. Conceitualmente, ambientes de geometria dinâmica são sistemas computacionais que proporcionam construções gráficas e manipulações espaciais de sólidos e curvas geométricos. Mais recentemente, a geometria dinâmica tem sido designada como geometria interativa (FERREIRA et al, 2012).

O professor que acredita que o aluno aprende Matemática através da memorização dos fatos, regras ou princípios transmitidos pelo professor ou pela repetição exaustiva de exercícios, também terá uma prática diferenciada daquele que entende que o aluno aprende construindo os conceitos a partir de ações reflexivas sobre materiais e atividades, ou a partir de situações problema e problematizações do saber matemático (FIORENTINI, 1995).

Segundo Isotani (2004) a Geometria Dinâmica (GD) pode ser entendida como a implementação computacional da geometria tradicional, aquela da régua-e-compasso. $\mathrm{O}$ termo dinâmico em oposição à estrutura estática das construções da geometria tradicional. Na GD, após o aluno realizar uma construção, ele pode alterar as posições dos objetos iniciais e o programa redesenha toda sua construção, preservando as propriedades originais.

Em função desta possibilidade de alterar objetos preservando-se a construção podemos dizer que a GD é uma geometria do tipo 1- construção, N-testes, enquanto a tradicional de régua e compasso é do tipo 1-construção, 1-teste. Esta é para nós, a grande vantagem da GD sobre a geometria tradicional, pois permite que o aluno teste conjecturas e procure descobrir propriedades (ISOTANI, 2004, p.319).

Segundo Barros(2000) a resolução de exercícios de GD requer raciocínio espacial e abstrato que exercita o aluno a visualizar estruturas 3D mentalmente. Desta forma, o aprendizado de GD tradicionalmente é baseado na resolução de exercícios, abstratos e aplicado.

Neste trabalho iremos utilizar o GeoGebra, disponível em (www.geogebra.org).

O GeoGebra é um software de matemática dinâmica que permite construir e explorar objetos geométricos e algébricos, interativamente. Foi desenvolvido pelo austríaco Markus Hohenwarter da Universidade de Salzburg, em 2001, e destina-se para o ensino de Geometria, Álgebra e Cálculo nas escolas de ensino básico (SANTIAGO et al, 2008). 
De acordo com Medeiros (2016), no GeoGebra tem-se a possibilidade de atribuir cor às construções, e a percepção da cor quando o sujeito entra em contato com o objeto, que neste caso, tem - se um objeto virtual, ele retira características do objeto, ou seja, daquilo que ele observa.

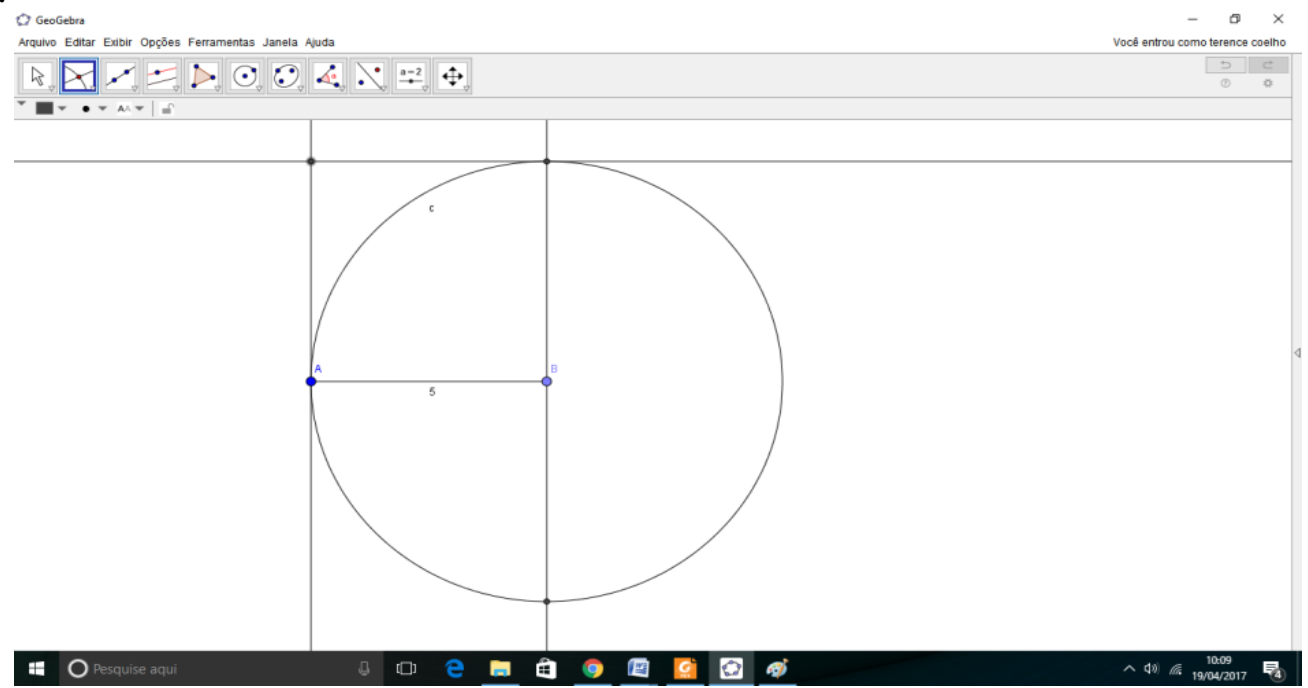

Figura 3 : Construção do quadrado no ambiente GeoGebra

Fonte: Arquivo pessoal

Para a utilização do programa para a aprendizagem dos conceitos geométricos, utiliza-se em três momentos: $1^{\circ}$ momento: Construção da figura, nesse momento é necessário o conhecimento dos elementos de geometria que compõem a figura, no caso da figura 13, tais como, segmento de reta, retas perpendiculares, retas paralelas, ponto de intersecção; $2^{\circ}$ momento: Identificação dos elementos e propriedades do quadrado, ângulos internos, ângulos externos, diagonais. Para esse momento direcionamos com perguntas aos alunos, Quanto medem os ângulos internos do quadrado? Quantas diagonais o quadrado possui? Qual o comprimento das diagonais? Quais as relações entre as diagonais? $3^{\circ}$ momento: Conclusão dos alunos sobre as observações sobre o quadrado.

Para responder aos questionamentos os alunos utilizam as ferramentas de construção

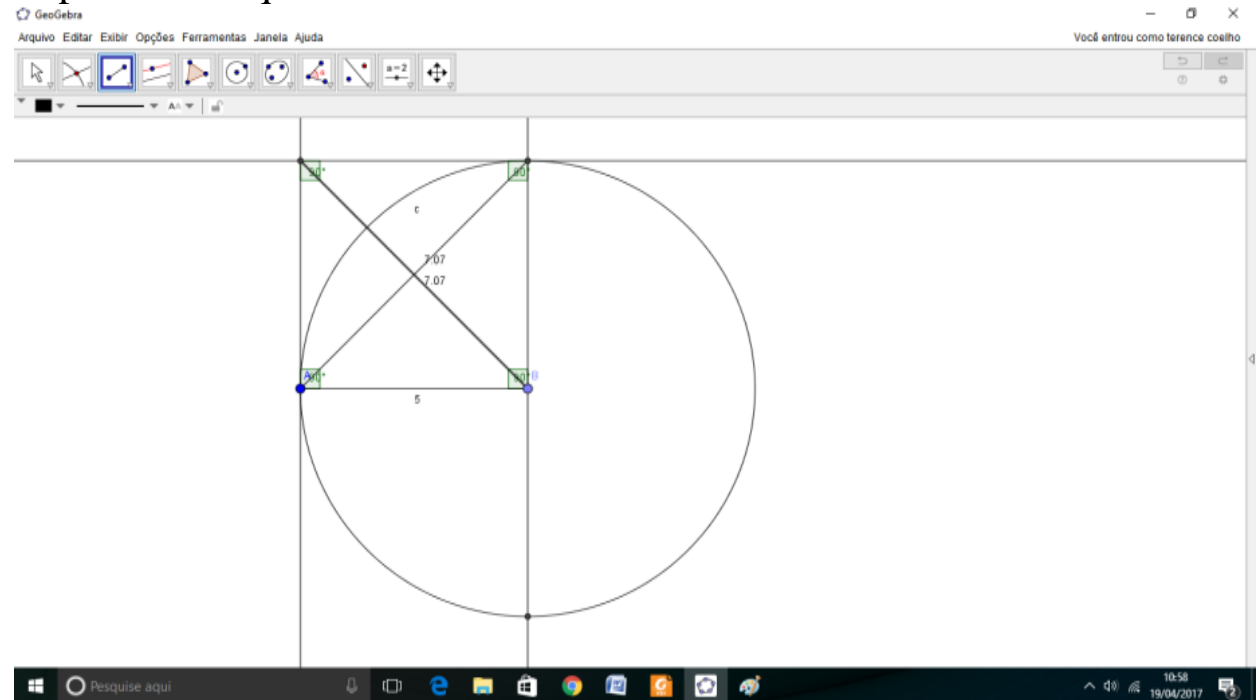

Figura 4: Construção do quadrado e verificação dos ângulos internos e comprimento das diagonais no GeoGebra.

Fonte: Arquivo pessoal

Para a observação das propriedades do quadrado e relação entre as diagonais, os estudantes precisam de direcionamentos para a investigação. 


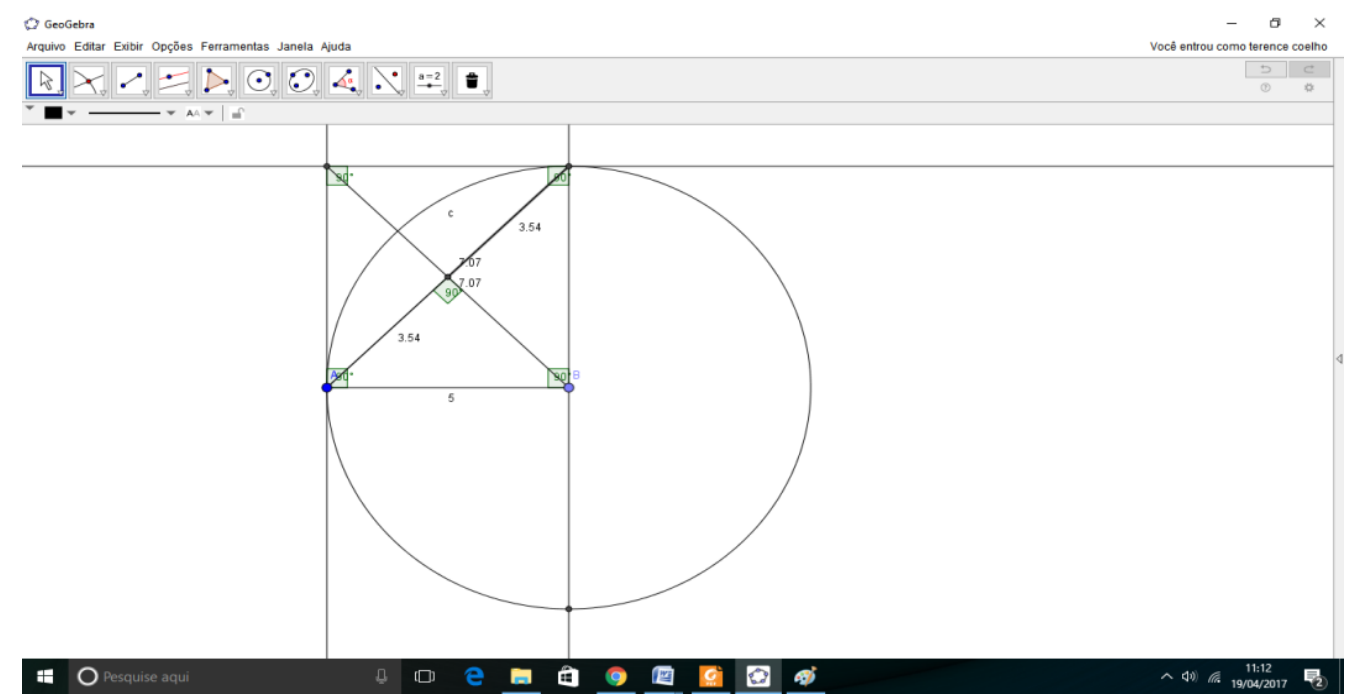

Figura 5: Construção do quadrado e a relação entre as diagonais e os ângulos internos Fonte: Arquivo pessoal As respostas obtidas foram:

- Os ângulos internos são congruentes e iguais a $90^{\circ}$;

- As duas diagonais são congruentes e iguais a 7,07;

- As diagonais são perpendiculares, se interceptam no ponto médio;

- As diagonais são as bissetrizes dos ângulos internos do quadrado.

\section{Arquitetura do Ambiente Virtual de Aprendizagem (AVA)}

Segundo Silva et al.(2014), os Ambientes Virtuais de Aprendizagem (AVA) são uma interface acessada através da internet, podendo ser personalizada de acordo com as demandas de cada curso (como também as demandas individuais) através das possibilidades que este recurso oferece, seja pela interação do aluno com o docente, com outros alunos e com as ferramentas didático-pedagógicas disponíveis.

Os AVA reúnem múltiplos recursos de armazenamento, de pesquisa e de comunicação disponíveis na internet, fomentando através de ferramentas a gestão do processo ensino-aprendizagem, para a distribuição de conteúdo, comunicação e interação entre os sujeitos do processo educativo (SILVA et al, 2014).

De acordo com Santos (2003), o AVA se desenvolve dentro do contexto de e- learning e que o conceito precisa ser problematizado, ou melhor, virtualizado. A aprendizagem mediada por AVA pode permitir que através dos recursos da digitalização várias fontes de informações e conhecimentos possam ser criadas e socializadas através de conteúdos apresentados de forma hipertextual, mixada, multimídia, com recursos de simulações.

Além do acesso e possibilidades variadas de leituras o aprendiz que interage com o conteúdo digital poderá também se comunicar com outros sujeitos de forma síncrona e assíncrona em modalidades variadas de interatividade: umum e um-todos comuns das mediações estruturadas por suportes como os impressos, vídeo, rádio e tv; e principalmente todos - todos, própria do ciberespaço (SANTOS, 2003).

Neste artigo, a busca pelo AVA como ambiente de aprendizagem se deve à possibilidade de fornecer aos estudantes suportes pedagógicos necessários para a elaboração 
da estratégia cognitiva de resolução dos problemas. Foi utilizada a plataforma Moodle que dispõe de ferramentas síncronas, como chats, onde a interação ocorre em tempo real, e as assíncronas, como fóruns, onde a interação ocorre em tempos diferentes.

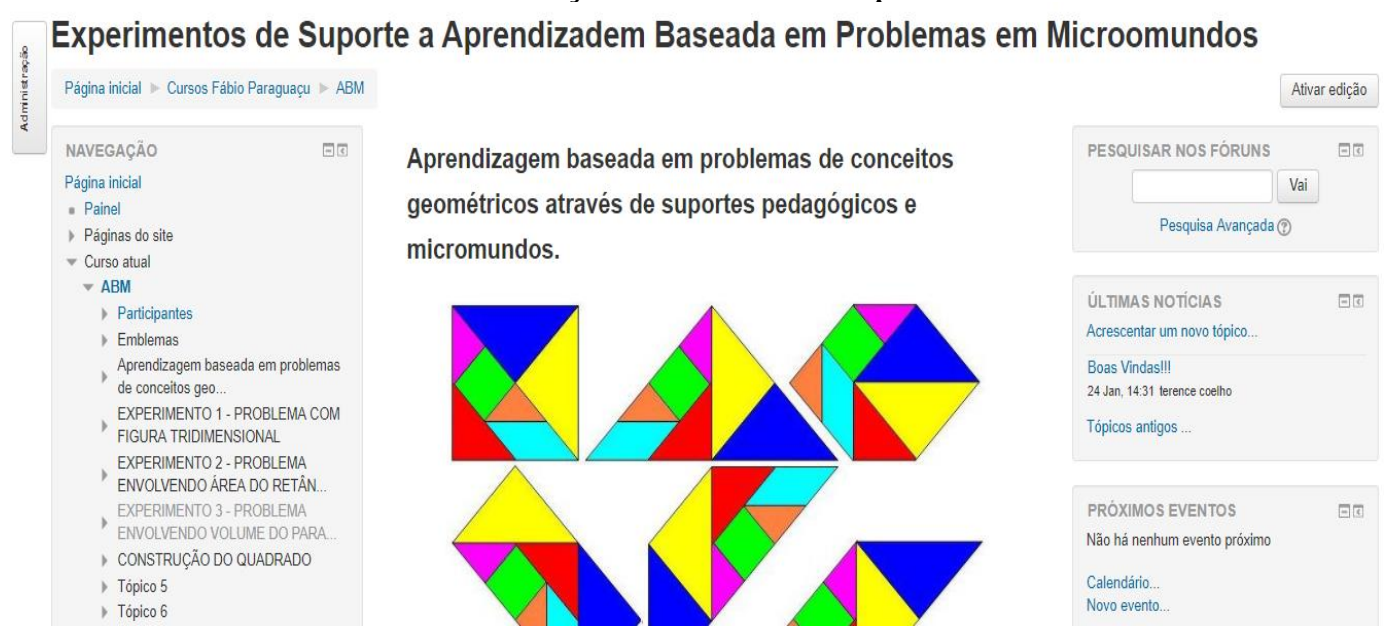

Figura 6: Ambiente virtual de aprendizagem com suportes pedagógicos

Fonte: Arquivo Pessoal

Sloczinski (2010) trata da autonomia do estudante em um ambiente virtual, da interação e das possibilidades de aprendizagem.

Quando aprendemos algo como, por exemplo, realizar uma operação matemática, interiorizamos o conceito e nos tornamos capazes de resolver outras atividades que envolvem esse conceito, implicando autonomia nesse aspecto, mas, se ainda não estamos capacitados para realizar determinadas tarefas ou resolver problemas relativos ao conceito, então precisamos de ajuda, de apoio (SLOCZINSKI, 2010, p.48).

A autora cita a Zona de Desenvolvimento Proximal (ZDP) de acordo com a teoria de Vygotsky, ou seja, quando o sujeito precisa de ajuda para realizar alguma atividade, ele se encontra na ZPD, entre o nível de desenvolvimento real (capacidade de realizar atividades de forma independente) e o nível de desenvolvimento potencial (capacidade de realizar atividades com a ajuda de outros mais experientes).

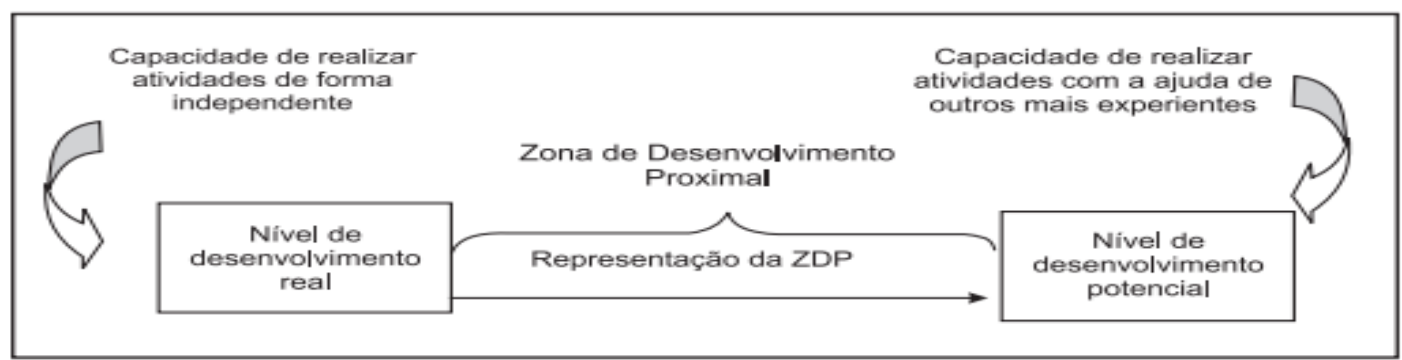

Figura 7: Zona de Desenvolvimento Proximal

Fonte: Sloczinski (2010)

Silva (2014) afirma que o termo ambiente virtual de aprendizagem (AVA) é designado para descrever o conjunto de sistemas necessários à gestão da aprendizagem online. Tais sistemas permitem a gestão de todos os processos, desde a criação do curso até a disponibilização do material didático ao aluno. Os AVAs são sistemas que fornecem suporte a diversos tipos de atividades realizadas pelo estudante e pelo professor, um conjunto de ferramentas que são usadas em diferentes situações dos processos de ensino e de aprendizagem. 
Dona aranha "Viúva Negra" tomava sol numa das faces de um cubo de $5 \mathrm{~cm}$ de aresta, quando ouviu: "dim, dim , dom !" Era uma de suas armadilhas. Bateu o olho no seu painel de controle e viu que era a armadilha Q.

"Viúva Negra" estava no ponto P. A pressa era tamanha que ela escolheu o menor caminho para se deliciar com o seu prato predileto: uma mosca. Se ela andou sobre as faces do cubo, qual é a distância que ela percorreu?Obs.: o ponto $\mathrm{P}$ está na face $\mathrm{ABCD}$ e o ponto $\mathrm{Q}$, na face $\mathrm{ABEH}$.

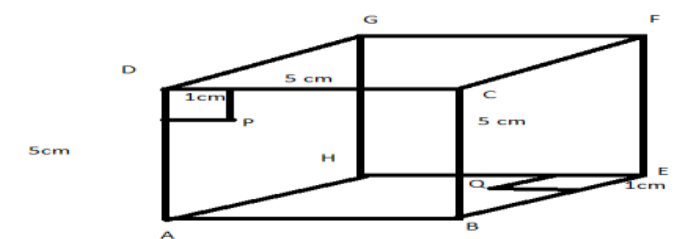

Figura 8: Experimento com figura geométrica tridimensional no AVA

Fonte: Arquivo Pessoal

O experimento, figura 8, representa um problema de geometria com uma figura tridimensional, os estudantes leram o problema e descreveram como resolveram o problema no fórum proposto na figura 9, "Como resolvo o problema".

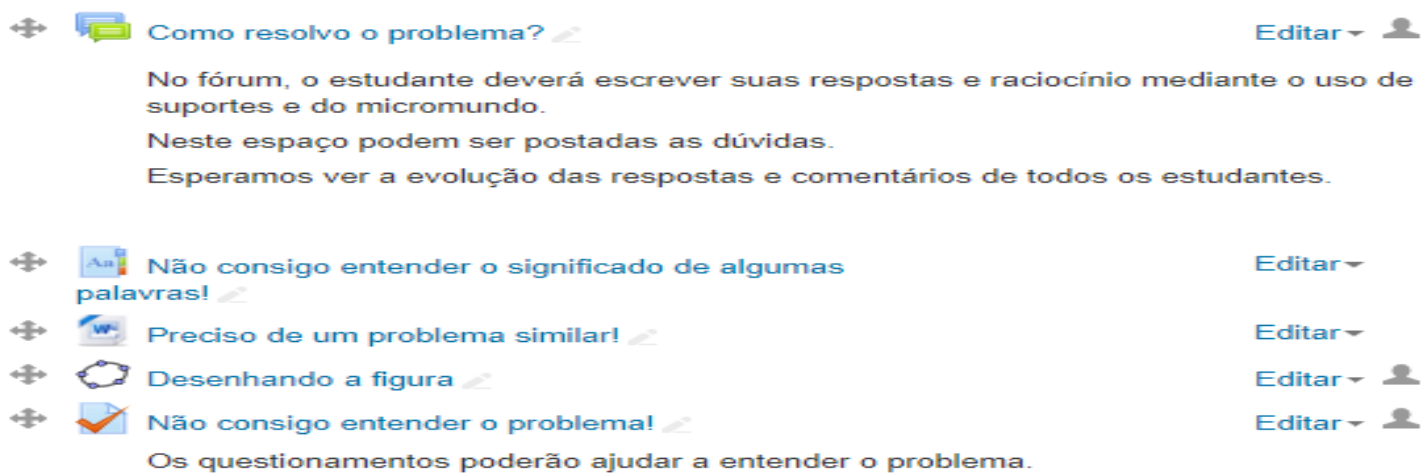

Figura 9: Suportes pedagógicos dispostos no AVA, baseados nas fases de Polya

Fonte: Arquivo pessoal

Foi proposto um Glossário das palavras desconhecidas pelos estudantes "Não consigo entender o significado de algumas palavras"; Um problema similar, que auxiliou o estudante com a identificação da incógnita e dos dados dos problemas "Preciso de um problema similar", o applet de geometria dinâmica, figura 10, "Desenhando a figura" e um questionário para interpretação e compreensão do problema "Não consigo entender o problema".

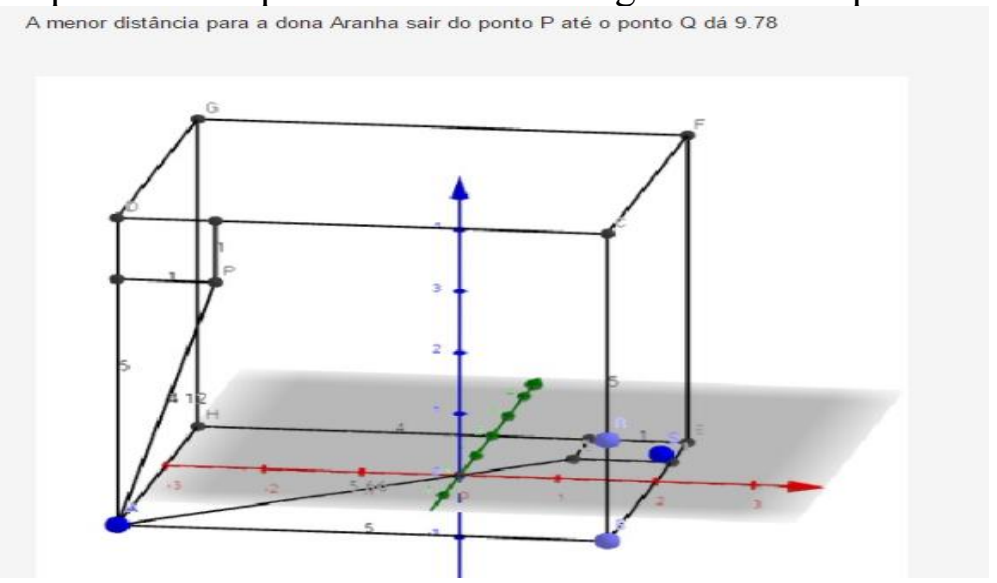

Figura 10: Representa a construção da figura geométrica no GeoGebra, como programa de aplicação no no moodle.

Fonte: Arquivo pessoal 
A figura 11 representa um cenário da modelagem computacional do ambiente de aprendizagem interligando cada fase de resolução de Polya e os suportes pedagógicos.

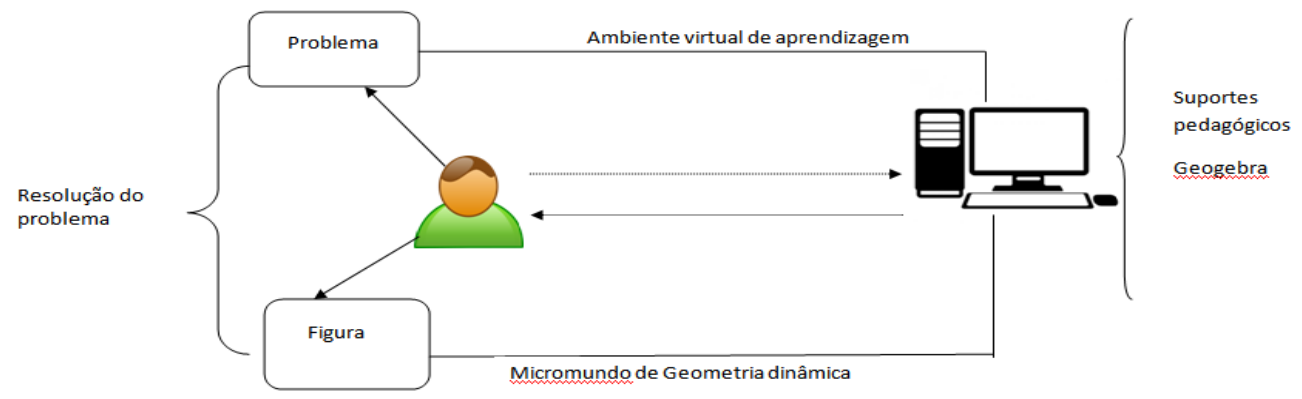

Figura 11: Cenário da modelagem do ambiente de aprendizagem

Fonte: Arquivo pessoal

\section{Metodologia}

A pesquisa foi realizada em uma escola pública de Ensino Fundamental e Médio, localizada na cidade de Maceió, Alagoas, em dois ambientes pedagógicos: na sala de aula presencial e no laboratório de informática, com 60 estudantes em duas turmas de $6^{\circ} \mathrm{s}$ anos.

A pesquisa é qualitativa e foi utilizado o Estudo de Caso, como metodologia de pesquisa, onde foram observados o processo de aprendizagem dos conceitos geométricos dos estudantes a partir das estratégias para resolução do problema proposto no Experimento, figura 8 .

Segundo Yin (2003), em geral, os estudos de caso representam a estratégia preferida quando se colocam questões do tipo "como" e "por que", quando o pesquisador tem pouco controle sobre os eventos e quando o foco se encontra em fenômenos contemporâneos inseridos em algum contexto da vida real. Pode-se complementar esses estudos de casos "explanatórios" com dois outros tipos - estudos "exploratórios" e "descritivos".

As fases desta pesquisa se concentraram no diagnóstico, implementação da aprendizagem baseada em problemas, estabelecimento do modelo em ambiente virtual de aprendizagem, análise de resultados.

No diagnóstico foi realizado um pré-teste nas duas turmas, onde se percebeu as dificuldades dos estudantes na resolução de problemas. Foram utilizados os Suportes Pedagógicos para auxiliar na elaboração de estratégias para resolução de problemas.

Foram utilizados, no laboratório de informática, o GeoGebra, para construção das figuras geométricas e compreensão da figura e um AVA, onde foram dispostos suportes para a interpretação do problema, através das ferramentas do moodle: Glossário, Questionário, Problemas similares e o fórum de socialização dos resultados.

\section{Resultados}

A tabela 1 expõe os resultados obtidos de acordo com as respostas dos estudantes nos estados inicial e final, nas fases de resolução de problemas e com a utilização dos suportes pedagógicos.

\begin{tabular}{|c|c|c|c|}
\hline Fases & $\begin{array}{ll}\begin{array}{l}\text { Resultado } \\
\text { (estado inicial) }\end{array} & 1 \\
\end{array}$ & $\begin{array}{l}\text { Suporte } \\
\text { pedagógico }\end{array}$ & Resultado 2(estado final) \\
\hline \multirow{2}{*}{$\begin{array}{l}\text { Compreensão } \\
\text { do Problema }\end{array}$} & \multirow{2}{*}{$\begin{array}{l}25 \% \text { dos } \\
\text { estudantes } \\
\text { conseguiram ler e } \\
\text { compreender. }\end{array}$} & Glossário & $40 \%$ dos estudantes conseguiram ler e compreender \\
\hline & & Questionamentos & $75 \%$ dos estudantes conseguiram ler e compreender \\
\hline Plano de ação & $\begin{array}{l}\text { Nenhum } \\
\text { estudante } \\
\text { elaborou um } \\
\text { plano de ação. }\end{array}$ & Problema Similar & $\begin{array}{l}80 \% \text { dos estudantes propuseram um plano de ação para } \\
\text { resolução do problema }\end{array}$ \\
\hline
\end{tabular}




\begin{tabular}{|l|l|l|l|l|}
\hline $\begin{array}{l}\text { Execução do } \\
\text { plano de ação }\end{array}$ & ----- & $\begin{array}{l}\text { Desenho da } \\
\text { Figura }\end{array}$ & $\begin{array}{l}80 \% \text { dos estudantes propuseram uma resposta, mas 5\% } \\
\text { conseguiram propor a resposta correta. }\end{array}$ \\
\hline Retrospecto & ----- & $\begin{array}{l}\text { Socialização de } \\
\text { resoluções }\end{array}$ & $\begin{array}{l}70 \% \text { dos estudantes conseguiram compreender a menor } \\
\text { distância }\end{array}$ \\
\hline
\end{tabular}

Tabela 1: resultados das respostas dos estudantes comparando os estados inicial e final com a utilização dos Suportes Pedagógicos.

\section{Considerações Finais}

A pesquisa foi realizada em duas turmas de $6^{\circ} \mathrm{s}$ anos do ensino fundamental anos finais. Foi observada a resolução do problema proposto no experimento 1(figura 9), através das fases propostas por Polya e foram utilizados os suportes pedagógicos para auxiliar no processo de aprendizagem.

O estudo de caso foi embasado no experimento, que foi um problema de geometria com sólidos geométricos. A perspectiva do experimento foi verificar como os estudantes iriam responder o problema e suas respostas em cada fase, bem como analisar o comportamento dos estudantes quando utilizavam o suporte.

Observa-se que no estado inicial 25\% (tabela1) dos estudantes não conseguiram ler e compreender o problema, pois desconheciam o significado de palavras do vocabulário matemático e não conseguiram verificar a incógnita, os dados e as condicionantes do problema. Com a utilização do glossário matemático, $40 \%$ dos estudantes conseguiram ler e compreender o problema. Com a utilização dos questionamentos sobre o problema, os estudantes releram o problema em busca das respostas às perguntas, favorecendo a compreensão e a interpretação do problema. Foi observada a evolução na aprendizagem dos estudantes na interpretação, mediante as respostas obtidas, $75 \%$ dos estudantes conseguiram interpretar o problema.

No estado inicial nenhum estudante conseguiu elaborar um plano de ação para resolver o problema, então foi utilizado o Problema Similar, que auxiliou na elaboração de um plano de ação. Foi verificada uma grande dificuldade em compreender a figura geométrica. Foi utilizado o GeoGebra como programa de aplicação do Moodle, sendo observado que $80 \%$ dos estudantes propuseram uma forma de resolver o problema de forma satisfatória, entretanto, $5 \%$ chegaram à resposta correta. Com a participação no fórum e socialização das respostas os estudantes fizeram um retrospecto das respostas e $70 \%$ conseguiram obter a menor distância, a solução correta.

Desta maneira observou - se que os resultados foram satisfatórios, que houve avanço na aprendizagem de conceitos geométricos através da resolução de problemas com o uso de suportes pedagógicos.

\section{Referências}

AUSUBEL, David P. Aquisição e Retenção de Conhecimentos: Uma Perspectiva Cognitiva. Plátano Edições Técnicas. Lisboa, 2001.

BARROS, Leilane Nunes. SANTOS, Eduardo Toledo. Um estudo sobre a modelagem do domínio de Geometria Descritiva para a construção de um Sistema Tutor Inteligente. In: XI Simpósio Brasileiro de Informática Educativa, p. 259-66, Maceió/AL, Nov, 2000.

BARROWS, Howard S, TAMBLYN, Robyn M. Problem-Based Learning - An Approach Medical Education. Springer Publishing Company. New York, 2010.

BAYER, Arno. LOBO, Joice da Silva. O Ensino de Geometria no Ensino Fundamental. In: ACTASCIENTIAE. V.6, n.1,JAN./JUN., 2004.

BRASIL. Parâmetros Curriculares Nacionais. Secretaria de Educação Fundamental. - Brasília: MEC/SEF, 1997.

FERREIRA, Emília B., Soares, Adriana B., Lima, Cabral. Matemáticas: Um estudo de caso sobre a geometria dinâmica e as pesquisas de campo com ambientes computacionais de ensino. In: Revista Brasileira de Informática na Educação, volume 20, número 3, 2012. 
FIORENTINI, Dário. Alguns modos de ver e conceber o Ensino da Matemática no Brasil. In: Revista Zetetiké, Ano 3, número 4, 1995.

GRAVINA, Maria Alice. Geometria Dinâmica Uma Nova Abordagem para o Aprendizado da Geometria. In: VII Simpósio Brasileiro de Informática na Educação, p.1-13, Belo Horizonte, Brasil, Nov, 1996.

ILLERIS, Knud. Teorias Contemporâneas da Aprendizagem (Org.). Porto Alegre: Penso, 2013.

ISOTANI, Seiji. BRANDÃO, Leônidas de Oliveira. Ferramenta de Avaliação no IGEOM. In: XV Simpósio Brasileiro de Informática na Educação (SBIE), 2004.

MEDEIROS, Margarete F. O Processo de Abstração Reflexionante na Construção de Conceitos Geométricos em Ambientes de Geometria Dinâmica. In: Revista Novas Tecnologias na Educação. V. 14 n.2. CINTED/UFRGS, 2016.

PERRENOUD, Philippe. Construindo Competências. In: Nova Escola, Brasil, setembro, PP 19-31, 2000.

POLYA, G. A arte de resolver problemas: um novo aspecto de método matemático, Rio de Janeiro: Interciência,1995.

PORTILHO, Evelise. Como se Aprende?Estratégias, Estilo e Metacognição.2ed. Rio de Janeiro, 2011. REISER, Brian. Scaffolding Complex Learning: The Mechanisms of Structuring and Problematizing Student Work. In: The Journal of the learning sciences,13(3), 273304, Lawrence Erlbaum Associates, Inc, 2004.

SANTIAGO, Lívia M. L.; ROCHA, Elizabeth M. LOPES, Josiane O. ANDRADE, Viviane Silva. MOREIRA, Marília M.; SOUSA, Tiago Gadelha; BARROSO, Acúrcio Magalhães; NETO, Hermínio Borges. Uso de Geogebra nas Aulas de Matemática: Reflexão Centrada na Prática. Artigo publicado em XIX Simpósio Brasileiro em Informática na Educação (SBIE), 2008.

SILVA, Alexandre José de Carvalho. Desenvolvimento de uma comunidade virtual para a inserção da metodologia blended learning na Educação Básica, no estado de Minas Gerais. Lavras: UFLA, 2014. Dissertação de Mestrado.

SILVA, Fernando Carlos Alves. PEREIRA, Geziel Alves. SOARES, Valdenir Maria Pereira. Ambientes Virtuais de Aprendizagem: O Uso das Tecnologias da Informação e Comunicação na Prática Pedagógica.In: Revista Itinerarius Reflectionis, volume 10, n.2, UFG/GO, 2014.

SLOCZINSKI, Helena. SANTAROSA, Lucila Maria Costi. "Como crescemos... Aprendemos tanto..." Construções sociocognitivas em distância, mediado pela Web. In: VALENTINI, Carla Beatriz. SOARES, Eliana Maria. Aprendizagem em Ambientes Virtuais (org.) - compartilhando idéias e construindo cenários. $2^{\text {a }}$. Ed. Caxias do Sul - RS: Educs, 2010.

VALENTE, Vânia Cristina Pires Nogueira. Ambiente Computacional interativo e adaptativo para apoio ao aprendizado de geometria descritiva. São Paulo: EPUSP, 2004.

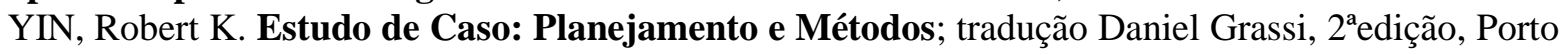
Alegre: Bookman, 2001. 